\title{
7
}

\section{Cataclysmic Variables}




\title{
AM HERCULIS BINARIES
}

\author{
K. BEUERMANN \\ Universitäts-Sternwarte Göttingen \\ Geismarlandstr. 11, D-37083 Göttingen, Germany
}

\begin{abstract}
AM Herculis binaries contain mass accreting magnetic white dwarfs which appear as bright X-ray sources in the ROSAT All Sky Survey. About 52 systems are presently known which allow detailed studies of the evolution of magnetic close binaries and of fundamental plasma-physical processes in the accretion region on the white dwarf.
\end{abstract}

\section{Introduction}

AM Herculis binaries are a subgroup of cataclysmic variables (CVs) which contain a mass losing late-type main-sequence star and an accreting magnetic white dwarf. Orbital periods range from $78 \mathrm{~min}$ to 4.6 hours with a further probable member of the class at 8.0 hours. The magnetic nature of the accreting white dwarf is manifested in a variety of ways, including the regular modulation of the optical and X-ray fluxes with superimposed flaring, the strongly polarized cyclotron emission, the occurrence of cyclotron lines in the optical/IR, and the Zeeman splitting of the Balmer absorption lines by typically several $100 \AA$. Both Zeeman splitting and the determination of the electron gyrofrequency from the spacing of the cyclotron lines, allow a determination of the field strength and to some extent also of the field structure. The latter determines the accretion flow, the location of the accretion spot(s), and, thereby, the overall appearance of the systems. Accreting matter of moderate density is shock-heated above the surface of the white dwarf and cools by hard X-ray bremsstrahlung and optical/IR cyclotron radiation. Dense filaments or blobs of matter, on the other hand, may penetrate into the photosphere. Their energy seems to be reverberated into the intense soft X-ray emission which is characteristic of AM Her stars. 


\section{Evolutionary Aspects}

The accretion geometry depends on whether the white dwarf rotates freely (DQ Her stars) or synchronously with the orbital period (AM Her stars). Among the physical processes which may be responsible for the synchronization, a particularly simple mechanism is the magnetostatic interaction of the magnetic moments $\mu_{1}$ and $\mu_{2}$ of primary and secondary, respectively (Campbell 1985). Synchronization takes place when the corresponding torque $G_{\text {sync }}$ exceeds the accretion torque $G_{\text {acc }}$ with $G_{\text {sync }} \sim \mu_{1} \mu_{2} / a^{3} \propto$ $P_{\text {orb }}^{-2}$ and $G_{\text {acc }} \simeq \dot{M} \Omega r_{\mathrm{L}}^{2} \propto P_{\text {orb }}^{1 / 3}$, where $a$ is the binary separation, $r_{\mathrm{L}}$ the distance between the inner Lagrange point and the white dwarf, $\Omega=2 \pi / P_{\text {orb }}$, and, for simplicity, the accretion rate $\dot{M}$ and the masses of the primary and the secondary were taken as constant (i.e., $r_{\mathrm{L}} \propto a \propto P_{\text {orb }}^{2 / 3}$ ). Equating the torques suggests as an order-of-magnitude estimate that synchronization takes place near $P_{\text {orb }} \sim 4$ hours for $\mu_{1} \sim 10^{34} \mathrm{G} \mathrm{cm}$ ( $\left.B \sim 30 \mathrm{MG}\right)$, an accretion rate $\dot{M} \sim 10^{16} \mathrm{~g} \mathrm{~s}^{-1}$, and an assumed magnetic moment of the secondary of $\mu_{2} \sim 10^{33} \mathrm{G} \mathrm{cm}^{3}(B \sim 100 \mathrm{G})$. Given the marked variations which $\dot{M}$ undergoes in individual systems, it is possible that a system which is synchronous at one time may break loose if $\dot{M}$ increases for a prolonged time. An estimate of the time scale for such variability is given by the period of harmonic oscillations of the magnetic axis about the line connecting the two stars. This period is of the order of $50 \mathrm{yrs}$ (Campbell 1985; Wickramasinghe \& Wu 1991; King \& Whitehurst 1991). Observational evidence for a secular change in the orientation of the white dwarf is scarce so far, with the possible exception of DP Leo (Beuermann \& Schwope 1994; Robinson \& Córdova 1994). The long-period magnetic nova V1500 Cyg $\left(P_{\text {orb }}=201 \mathrm{~min}\right)$ lost synchronism in the common-envelope phase of the eruption and is currently re-establishing it on a time scale of $170 \mathrm{yrs}$ (Schmidt et al. 1994). Studies of this system can provide important information about the mechanism of synchronization. Unfortunately, V1500 Cyg is faint and the field strength of the white dwarf is uncertain. BY Cam with $P_{\text {orb }}=202 \mathrm{~min}$ is another system which is seemingly slightly out of synchronism (Silber et al. 1992; Piirola et al. 1994). It will also be worthwhile to search for the degree of synchronism in the other 9 systems with still longer periods of which 7 are newly discovered with ROSAT. Presently, the systems with the longest periods are RX J1313-32 $\left(P_{\text {orb }}=255 \mathrm{~min}\right), \mathrm{RX} \mathrm{J0203+29}\left(P_{\text {orb }}=275 \mathrm{~min}\right)$ and RX J0515+01 $\left(P_{\text {orb }}=480 \mathrm{~min}\right)$ (Beuermann \& Schwope 1994; Garnavich et al. 1995; Shafter et al. 1995; Walter et al. 1995).

Since DQ Her stars dominate among the known longer-period magnetic CVs and AMHer stars among the short-period ones, King et al. (1985) suggested that DQ Her stars evolve into AM Her stars once they become synchronized. This appears to be a viable hypothesis, save for the fact that 


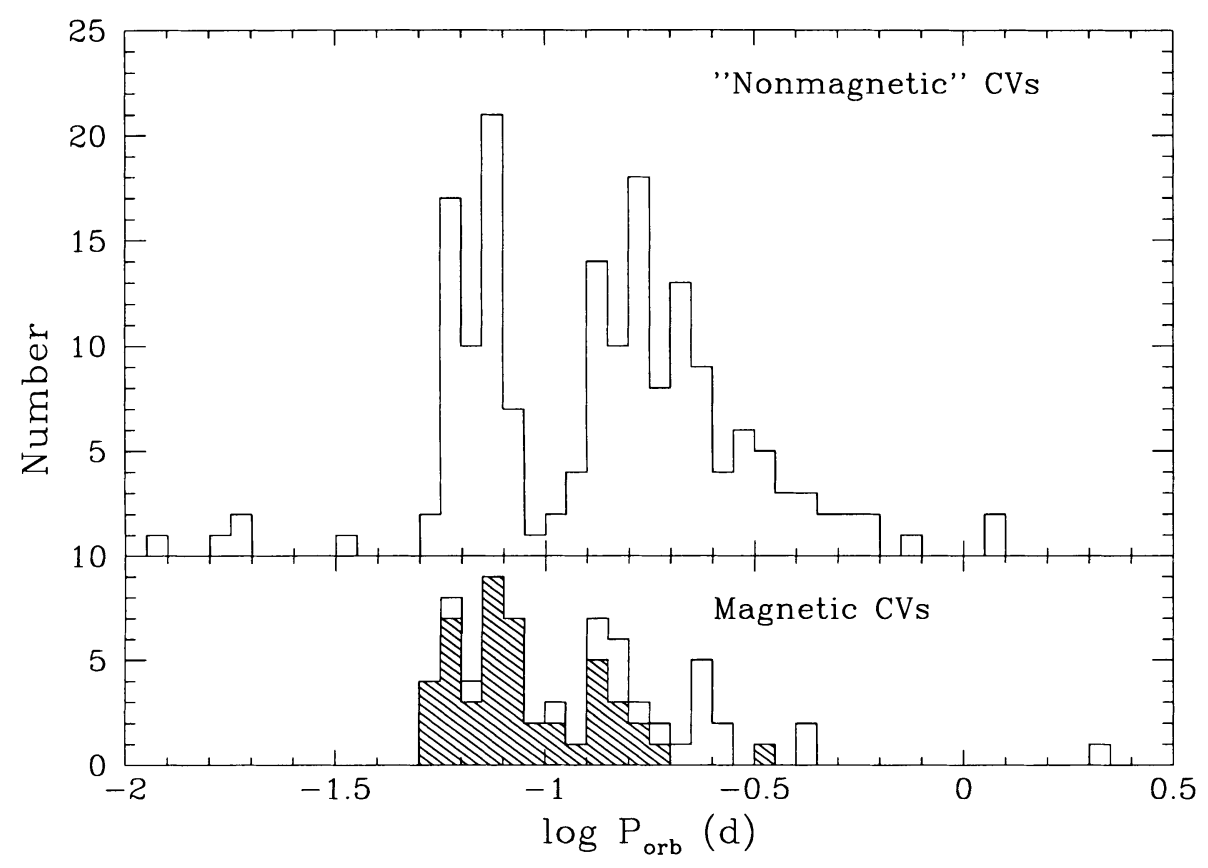

Figure 1. Orbital-period distribution of non-magnetic and magnetic CVs. The shaded histogram in case of the magnetic CVs denotes the AM Herculis binaries, the open part the DQ Her stars. Both diagrams indicate a paucity of systems with periods between 2 and 3 hours around $\log P_{\text {orb }}$ (days) $=-1$.

most DQ Her stars seem to have lower polar-field strengths than AM Her stars. Nevertheless, the space density of DQ Her stars is comparable to that of long-period AM Hers $\left(n \sim 710^{-8} \mathrm{pc}^{-3}\right)$ and at least some DQ Her stars may be expected to ultimately become synchronized. Several new ROSATdiscovered DQ Her stars with extremely soft X-ray spectra similar to those of AM Her stars (Mason et al. 1992; Haberl et al. 1994) may be progenitors of AMHer stars. The field strength of RX J0751+14 = RE $0751+14, B \simeq$ 8-18 MG (Piirola et al. 1993), is comparable to those of white dwarfs in some AM Her stars.

The principal impact of the ROSAT mission on the study of AM Herculis binaries was to substantially increase the number of known systems to beyond 50 . This allows to proceed from the study of individuals to the study of the properties of the class.

Fig. 1 shows the distribution of orbital periods $P_{\text {orb }}$ of magnetic CVs along with that of non-magnetic CVs (Ritter \& Kolb 1993). A CV appears on the long-period side of the distribution when the secondary first gets in contact with its Roche surface and evolves towards lower orbital periods 
by loss of angular momentum until the secondary becomes degenerate at $P_{\text {orb }} \simeq 80 \mathrm{~min}$. A pronounced feature is the famous 'period gap' between 2 and 3 hours. At $P_{\text {orb }}>3$ hours, a CV loses angular momentum presumably by 'magnetic braking', i.e., by a stellar wind driven from the magnetosphere of the secondary (Verbunt \& Zwaan 1981), in addition to gravitational radiation which acts on all systems. The conventional explanation of the period gap involves cessation of magnetic braking at $P_{\text {orb }} \simeq 3$ hours when the secondary becomes fully convective and loses its magnetic activity. The associated reduction in $\dot{M}$ causes the secondary to relax to thermal equilibrium and recede from its Roche surface. Mass transfer resumes when gravitational radiation has re-established contact at $P_{\text {orb }} \simeq 2$ hours.

It has been suggested that the magnetic configuration of AM Her stars prevents the wind loss, causing them to evolve merely by gravitational radiation (Wickramasinghe \& $\mathrm{Wu} 1994$ ) in which case there would be no gap. The observational situation seems to refute that suggestion. If we define the gap as extending from $\log P_{\text {orb }}$ (days) $=-0.90$ to -1.05 (2.1 to 3.0 hours) then the numbers below the gap and in the gap, and the corresponding ratios are $57,7,0.12 \pm 0.05$ for the non-magnetic systems and 30 , $5,0.17 \pm 0.08$ for the AM Her stars, respectively. While these two ratios are not significantly different, there are clearly much fewer magnetic systems above the gap than non-magnetic ones. This holds for AM Her stars alone and for all magnetic systems. One reason is that a typical CV will appear at $P_{\text {orb }} \sim 8$ hours while a typical magnetic CV synchronizes only when $P_{\text {orb }} \sim 4$ hours. Nevertheless, the presence of a gap indicates that the same process which causes the gap in non-magnetic CVs is acting also in magnetic ones.

The distributions in Fig. 1 are not free from selection effects in the sense that they do not represent the true space densities at a given period. Longer-period systems are generally drawn from a larger volume but not as large as may be expected from their luminosities because absorption of the soft X-ray emission causes a systematic loss of more distant systems. In summary, magnetic and non-magnetic CVs seem to evolve under the same angular-momentum loss processes but differences in the relative efficiencies of these processes for the two subclasses can not be excluded.

\section{Magnetic Field of the White Dwarf}

The strength and structure of the white-dwarf magnetic field determine not only the evolution of a system but also the accretion geometry and the radiative properties. Part of the luminosity is released as cyclotron radiation which displays the higher harmonics of the electron gyrofrequency as severely broadened emission lines from the $\sim 10^{8} \mathrm{~K}$ post-shock plasma. 

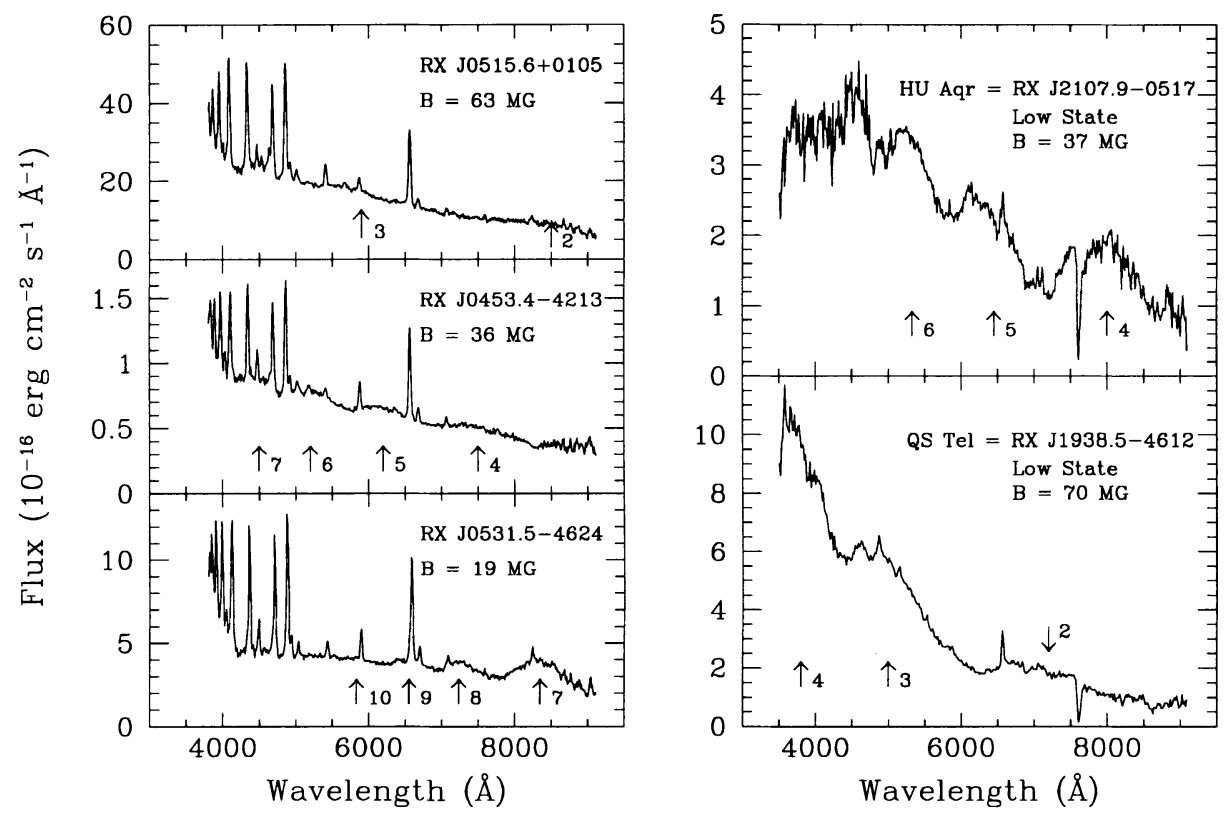

Figure 2. Examples of cyclotron lines in five AM Herculis binaries newly discovered with ROSAT. Line positions, harmonic numbers, and field strengths derived from their separations are indicated.

After minor special-relativistic corrections, the separation of these lines directly measures the field strength $B_{\text {cyc }}$ in the accretion spot. Fig. 2 shows examples of cyclotron lines in five of the AM Herculis binaries newly discovered with ROSAT (Burwitz et al. 1994; Reinsch et al. 1994; Schwope et al. 1993a, 1994; Shafter et al. 1994).

Cool matter surrounding the accretion spot may lead to Zeeman lines in the cyclotron quasi-continuum. This effect measures the field strength $B_{\mathrm{h}}$ in a gaseous halo around the accretion spot and possibly at some height above the photosphere. Finally, photospheric Zeeman lines may be detected if accretion ceases or the spot is hidden behind the white dwarf. In this case, a flux-weighted mean field $\bar{B}_{\text {phot }}$ over the visible hemisphere is measured. In lucky circumstances, a combination of such measurements provides insight into the field structure of the white dwarf. We may expect $B_{\mathrm{h}} \simeq B_{\text {cyc }} \simeq$ $B_{\text {pole }}$ because emission and absorption occur near one of the poles and nearby in space; furthermore, in a dipole geometry $B_{\text {pole }}$ would exceed $\bar{B}_{\text {phot }}$ by a factor of up to 2 . Surprisingly, however, in some systems $\bar{B}_{\text {phot }}$ is similar to $B_{\text {cyc }}$ (or $B_{\mathrm{h}}$ ) or even significantly exceeds it, a result which indicates substantial deviations of the surface field from a dipole geometry.

Table 1 provides an overview of currently available field measurements of the white dwarfs in 21 AM Her stars. Field strengths for the main accretion 
region cluster around $30 \mathrm{MG}$ with a standard deviation of $15 \mathrm{MG}$. There are no field strengths below $\sim 10 \mathrm{MG}$ which probably indicates that even short-period systems do not synchronize below this value. There are also no field strengths in the main accretion spot in excess of $61 \mathrm{MG}$. This is surprising because single white dwarfs show a much broader distribution which extends to beyond $500 \mathrm{MG}$ (Chanmugam 1992). A viable hypothesis for the absence of high-field magnetic CVs involves a strong wind which couples onto the white-dwarf field lines and cause rapid evolution of such systems with a correspondingly small space density and chance of discovery (Hameury et al. 1988).

For some systems, two values are given for $B_{\text {cyc }}$. In these cases, two systems of cyclotron lines were observed which refer to two accretion regions. If these are located essentially opposite to each other, field strengths different by a factor of $\sim 2$ are incompatible with a dipole. In addition, there is evidence for non-dipolar fields also in other systems in which either no cyclotron lines are observed (e.g., because of excessive temperature broadening) or the second pole is hidden behind the white dwarf. E.g., BL Hyi displays pronounced Zeeman absorption lines in the cyclotron continuum which indicate a field strength of $12 \mathrm{MG}$ in the accretion region. In low states, however, shallow Zeeman absorption lines appear which indicate a mean photospheric field of $22 \mathrm{MG}$ with a substantial spread in field strength (Schwope et al. 1995). This is impossible in a dipole geometry. A somewhat less extreme example is MR Ser with a field of $24 \mathrm{MG}$ in the main accretion spot and a mean photospheric field of $27 \mathrm{MG}$. In this case, it is noteworthy that all individual non-degenerate Zeeman components of $\mathrm{H} \alpha \sigma^{-}$and $\mathrm{H} \alpha \sigma^{+}$are detected which imply a field spread of less than $1 \mathrm{MG}$ over the visible hemisphere (Schwope et al. 1993b). Assuming a field structure of aligned dipole and quadrupole components (or a dipole offset along its axis) implies field strengths of $\sim 60 \mathrm{MG}$ at the second pole. Representing the observed spectra by synthetic Zeeman spectra for such configuration was quite successful for MR Ser but less so for BL Hyi, suggesting perhaps a more complex field structure in the latter (Schwope et al. 1993b, 1995).

Accepting direct and indirect evidence, there are 9 systems with significantly different field strengths at two 'poles'. In all cases, the main accretion spot is located at the low-field 'pole'. For neutron stars, Romani (1993) suggested that accretion may cause the horizontal component of the field to be submerged and carried away from the pole. Unfortunately, no quantitative treatment exists and the applicability to white dwarfs remains open because the total accreted mass is uncertain. Also, nova explosions may counteract submersion. Alternatively, the non-dipolar field structure could be a property of the internal field generating process in the progenitor star and the magnetic orientation of the white dwarf a consequence of the mechanism 
TABLE 1. Field strengths of magnetic white dwarfs in cataclysmic variables. $B_{\text {cyc,1 }}$ and $B_{\mathrm{cyc}, 2}$ refer to field strengths obtained from the spacing of cyclotron lines, $B_{\mathrm{h}}$ to the Zeeman effect in the cool halo of the accretion spot, and $\bar{B}_{\text {phot }}$ to the flux-weighted mean photospheric field from Zeeman lines.

\begin{tabular}{|c|c|c|c|c|c|c|}
\hline System & $\begin{array}{c}P_{\text {orb }} \\
(\min )\end{array}$ & $\begin{array}{l}B_{\text {cyc }, 1} \\
(\mathrm{MG})\end{array}$ & $\begin{array}{l}B_{\text {cyc }, 2} \\
(\mathrm{MG})\end{array}$ & $\begin{array}{c}B_{\mathrm{h}} \\
(\mathrm{MG})\end{array}$ & $\begin{array}{l}\bar{B}_{\text {phot }} \\
\text { (MG) }\end{array}$ & References \\
\hline EF Eri & 81 & & & 13 & & 1 \\
\hline DP Leo & 90 & 31 & 59 & & & $2,3,4$ \\
\hline RX J1149+28 & 90: & 43 & & & & 5 \\
\hline RX J1957-57 & 99 & & & 14 & & 6 \\
\hline VV Pup & 100 & 31 & 54 & & & 3,7 \\
\hline V834 Cen & 101 & 23 & & 23 & 22 & 8,9 \\
\hline RX J0453-42 & 102: & 36 & & & & 10 \\
\hline MR Ser & 113 & 24 & & 24 & 27 & 11 \\
\hline BL Hyi & 114 & & & 12 & 22 & 12 \\
\hline ST LMi & 114 & 12 & & & 19: & 13,14 \\
\hline EK UMa & 114 & $35: / 47:$ & & & & 15 \\
\hline AN UMa & 115 & 29 & & & & 3,16 \\
\hline RX J2107-05 & 125 & 35 & & & & 17 \\
\hline EU Cnc & 125 & 42: & & & & 18,19 \\
\hline UZ For & 126 & 53 & $75(113)$ & & & 20 \\
\hline RX J0531-46 & 133 & 19 & & & & 21 \\
\hline RX J1938-46 & 140 & 47 & $70(80)$ & & & 22 \\
\hline AM Her & 186 & 14 & & & 13 & 23,24 \\
\hline BY Cam & 202 & 28 & & & & 3,16 \\
\hline QQ Vul & 222 & 36: & & & & 3 \\
\hline RX J0515+01 & 480 & 61: & & & & 25 \\
\hline
\end{tabular}

(1) Östreicher et al. 1990, (2) Cropper et al. 1990a, (3) Schwope 1991, (4) Cropper \& Wickramasinghe 1993, (5) Schwope (in prep.), (6) Thomas et al. (in prep.), (7) Wickramasinghe et al. 1989, (8) Schwope \& Beuermann 1990, (9) Ferrario et al. 1992, (10) Burwitz et al. 1995, (11) Schwope et al. 1993b, (12) Schwope et al. 1995, (13) Schmidt et al. 1983, (14) Ferrario et al. 1993, (15) Cropper et al. 1990b, (16) Cropper et al. 1988, (17) Schwope et al. 1993a, (18) Pasquini et al. 1994, (19) This work, (20) Schwope et al. 1990, (21) Reinsch et al. 1995, (22) Schwope et al. 1994, (23) Young et al. 1981, (24) Bailey et al. 1991, (25) Shafter et al. 1995.

of synchronisation (Wu \& Wickramasinghe 1993).

In summary, our knowledge of the field structure in magnetic CVs is still utterly incomplete but improving. In the future, high-sensitivity spectropolarimetry of the cyclotron radiation in high accretion states and of Zeeman absorption spectra in low states promise to yield interesting results. Availability of the polarization spectra provides additional information which is needed to distinguish between different possible field geometries (Putney \& Jordan 1994). 


\section{Accretion Scenarios}

In AM Herculis binaries, the magnetic field of the white dwarf prevents the formation of an accretion disk. After leaving the inner Lagrange point $L_{1}$, the accretion stream penetrates into the field along a quasi-ballistic trajectory until equality of ram pressure and magnetic pressure is reached. We know very little about the plasma-dynamical processes in the magnetosphere which cause break-up of the stream and coupling onto near-polar field lines (Hameury et al. 1986). Observationally, emission line measurements delineate the hot and partially photoionized section of the stream close to the white dwarf, but not the distant cooler sections, including that between $L_{1}$ and the stagnation point. Break-up of the stream probably determines the density distribution of the matter and thereby the spatial and temporal distribution of the mass flow rate arriving at the white-dwarf surface. Additional information on the density and ionization structure of the stream in the outer magnetosphere can be obtained from X-ray and IR absorption measurements at the orbital phase when the stream passes in front of the hot spot on the white dwarf (e.g., Watson et al. 1989).

Standard theory (Lamb \& Masters 1979; King \& Lasota 1979) assumes a stationary flow of matter $\dot{m}$ (in $\mathrm{g} \mathrm{cm}^{-2} \mathrm{~s}^{-1}$ ) which is shock-heated close to the surface of the star to $\sim 10^{8} \mathrm{~K}$; subsequently, the plasma cools by bremsstrahlung and cyclotron radiation until is settles onto the photosphere. Since part of the emission will be intercepted by the white dwarf, a third component will be due to reprocessed radiation. Directly below the shock, the effective temperature of the heated photosphere may exceed $\sim 310^{5} \mathrm{~K}$ if the shock height is small; the reprocessed flux then emerges at soft X-ray energies. If, on the other hand, the shock height in units of the white-dwarf radius is large, $h / R_{\mathrm{wd}} \sim 0.1$, as expected for low mass flow rates, the irradiated fraction of the white dwarf surface becomes large, too, $f \simeq h / 2 R_{\mathrm{wd}} \sim 0.05$. The temperature drops to $<10^{5} \mathrm{~K}$, and the emission peak moves into the UV. Beaming of the cyclotron emission perpendicular to the field and, therefore, parallel to the surface adds to a wider spread of the irradiation. Gänsicke et al. (1995) have studied the energy balance in AM Herculis itself and found that bremsstrahlung and cyclotron irradiation can quantitatively account for the UV flux of the large $(f \sim 0.08)$ heated spot, both in the low and in the high state. Hence, in AM Her this reprocessed component seems to emerge primarily in the UV and not in the soft X-ray regime. In other systems, however, this component may be irretrievably submerged in the accretion-produced soft X-ray emission.

Observationally, most AM Herculis stars are spectacular soft X-ray sources, but the soft $\mathrm{X}$-ray luminosity is much too large to be due to reprocessing. For years, this so-called 'soft X-ray problem' has plagued theoreticians and 


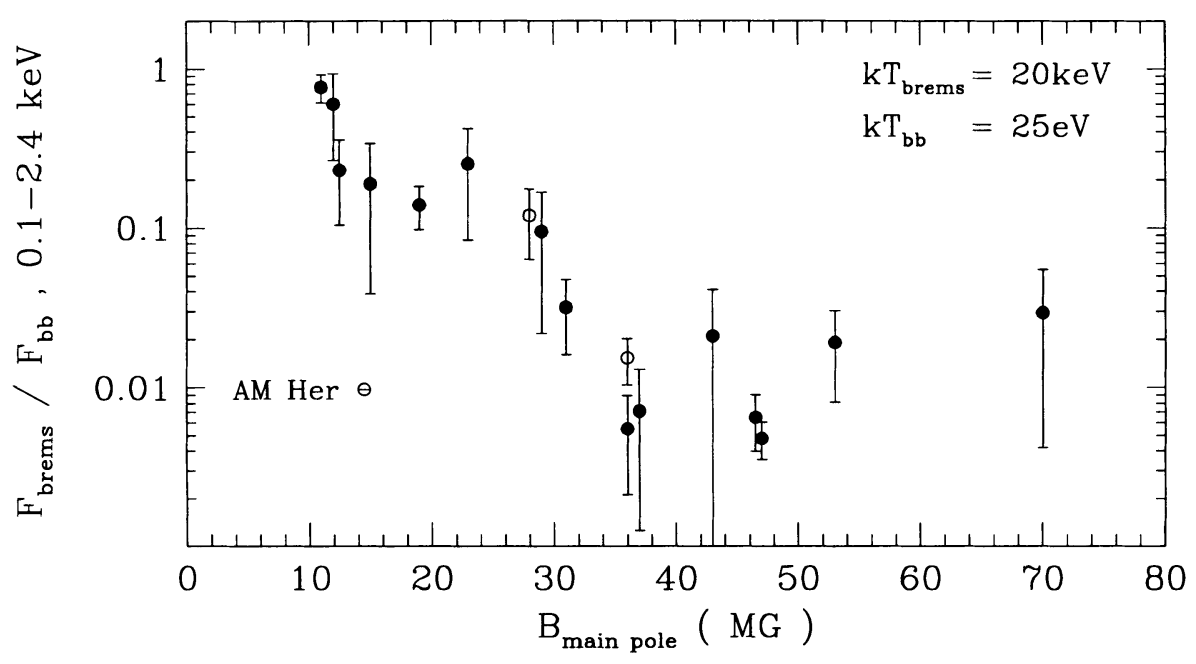

Figure 3. Ratio of the unabsorbed energy fluxes $F_{\text {brems }}$ and $F_{\mathrm{bb}}$ in the ROSAT (0.1-2.4 keV) window as a function of field strength in the accretion spot. Bremsstrahlung is increasingly suppressed at high field strengths. Filled and open circles refer to short-period and long-period ( $P_{\text {orb }}>3$ hours) systems, respectively.

observers alike until Kuijpers \& Pringle (1983) and Morfill et al. (1984) suggested that sufficiently dense blobs of matter might transfer their kinetic energy to sub-photospheric layers and heat the photosphere from below. Short time-scale fluctuation studies of soft and hard X-rays indicate that these two components are not correlated (e.g., Beuermann et al. 1991) which argues against an origin of the soft $X$-rays from reprocessing of the observed hard X-ray component. On the other hand, Van Teeseling et al. (1994) showed that the EXOSAT grating spectrum of AM Her is not consistent with an undisturbed hot LTE atmosphere, but is much better fitted by an irradiated atmosphere or an atmosphere heated by more diffuse blobs which disperse their energy at small optical depths. Hence, the hydrodynamics and the radiative transfer are certainly more complex than suggested by the original Kuijpers \& Pringle picture. The actual accretion region is probably structured with a wide range of co-existing mass flow rates. Cyclotron emission, hard X-ray bremsstrahlung and soft X-ray emission are probably due to different regimes in the mass flow rates of order $\sim 10^{-2}, \sim 1$, and $\sim 100 \mathrm{~g} \mathrm{~cm}^{-2} \mathrm{~s}^{-1}$, respectively (e.g., Beuermann et al. 1987).

The large number of AMHer stars observed with ROSAT has, for the first time, allowed a systematic study of the emission properties as a function of system parameters. Fig. 3 shows the ratio of the bremsstrahlung to quasi-blackbody fluxes as a function of field strength in the accretion 


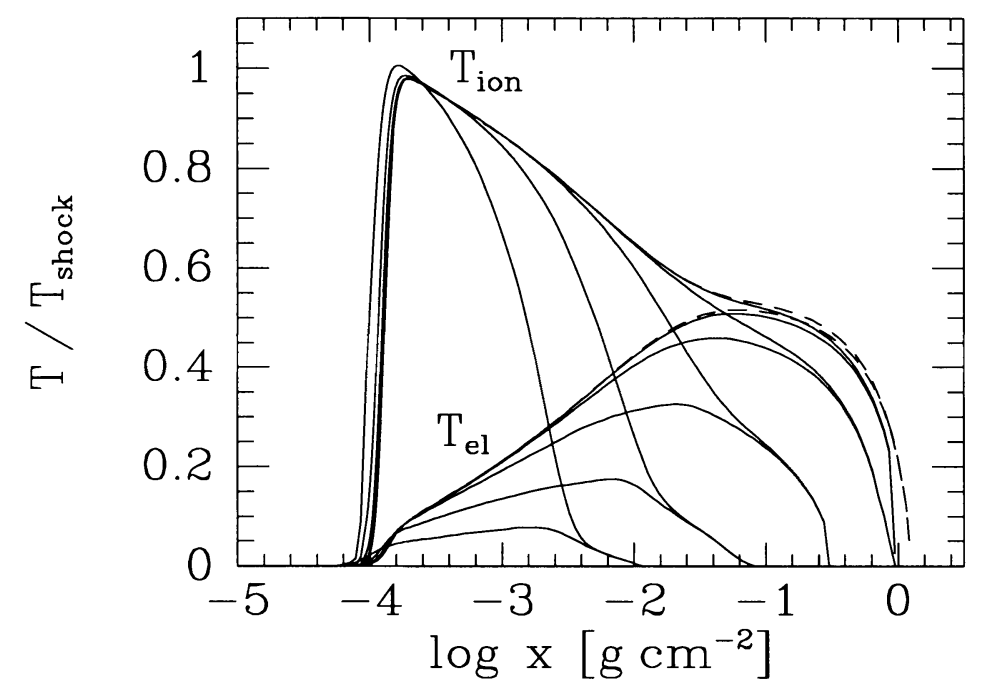

Figure 4. Two-fluid temperature structure of the shocked accretion flow for $B=30 \mathrm{MG}$ and mass flow rates of $\dot{m}=0.01$ to $100 \mathrm{~g} \mathrm{~cm}^{-2} \mathrm{~s}^{-1}$ (solid lines, from bottom to top). For comparison, the pure bremsstrahlung case for zero field and/or high mass flow rates is shown (dashed lines). Abscissa is column density of a pure hydrogen plasma, ordinate is temperature in units of $T=(3 / 16)(\mathrm{m} / k) v_{\mathrm{ff}}^{2}$. The flow enters from the left and, for each set of curves, the drop to zero temperature indicates the location of the stellar photosphere.

spot. $F_{\text {brems }}$ and $F_{\mathrm{bb}}$ are the fluxes in the ROSAT $0.1-2.4 \mathrm{keV}$ band corrected for interstellar absorption (fluxes 'at the source'). The flux ratio is seen to decrease systematically with field strength, indicating a suppression of bremsstrahlung and/or a preference of soft X-ray emission at high field strengths. Suppression is an obvious choice because cyclotron emission increases with $B$ and dominates over bremsstrahlung. Preference of soft $X$-ray emission may also occur because the mass flow rate in a magnetic funnel scales as $\dot{m}=\rho v \propto B$. Why AM Her, the supposed 'prototype of the class', avoids the general trend remains to be clarified.

Time-dependent radiation-hydrodynamical calculations are required to take full advantage of the plasma-diagnostic information content of the available observations. Such calculations which account for the fully angledependent and frequency-dependent radiative transfer of the cyclotron radiation are not yet available. As a step towards this goal, Woelk (1995) has solved the 1-D stationary two-fluid hydrodynamic equations together with the full radiative transfer for a constant field. His solutions are applicable for pill-box shaped accretion regions (i.e. not for tall columns which require 2 -D radiative transfer). As an example of his results, Fig. 4 shows the electron and ion temperatures in the post-shock region for $B=30 \mathrm{MG}$ and mass flow rates between $\dot{m}=0.01$ and $100 \mathrm{~g} \mathrm{~cm}^{-2} \mathrm{~s}^{-1}$, along with the pure 
bremsstrahlung solution which is the limiting case for zero field and also for very high $\dot{m}$. With decreasing mass flow rate (and thereby decreasing post-shock density), cyclotron cooling reduces the electron and the collisionally coupled ion temperatures far below the Hugoniot temperature. At the same time, the shock height $x$ (in $\mathrm{g} \mathrm{cm}^{-2} \mathrm{~s}^{-1}$ ) collapses to a small fraction of the bremsstrahlung case. The geometric shock height $h$ (in $\mathrm{cm}$ ), however, still increases with decreasing $\dot{m}$ but less so than $h \propto \dot{m}^{-1}$ which is the expected increase for cooling by bremsstrahlung alone. The calculated spectra yield a smooth cyclotron continuum for the higher $\dot{m}$ values, whereas cyclotron lines appear for small $\dot{m}$. Bremsstrahlung originates mainly from the high- $\dot{m}$ regions. At low $\dot{m}$, the solutions connect to the bombardment case studied earlier by Woelk \& Beuermann (1992, 1993).

In summary, we are beginning to understand the relevant plasma physics of the accretion spot, although many questions relating, e.g., to the internal structure of the spot or to the stability of the shock solutions remain open. Our knowledge of the processes in the magnetosphere of the white dwarf are still fragmentary at best.

\section{Conclusions}

The main impact of the ROSAT mission on the field lies in the substantial increase in the number of recognized magnetic CVs. We can now proceed from the study of individuals to the study of the class, use these systems as plasma laboratories, investigate the magnetic-field structure of compact stars, and learn more about the evolution of cataclysmic variables.

Acknowledgements. I thank A.D. Schwope, Potsdam, for some of the data in Table 1, and V. Burwitz and U. Woelk for preparing Figs. 1-3 and Fig. 4, respectively. I enjoyed interesting discussions with these colleagues and with B. Gänsicke, K. Reinsch, and A. van Teeseling, and acknowledge useful comments on the manuscript. This work was supported in part by the DARA under project number 500R9210.

\section{References}

Bailey, J., Ferrario, L. \& Wickramasinghe, D.T. 1991, MNRAS 251, 37P

Beuermann, K. \& Schwope. A.D. 1994, ASP Conf. Ser. 56, p. 119

Beuermann, K., Stella, L. \& Patterson, J. 1987, ApJ 316, 360

Beuermann, K., Thomas, H.-C. \& Pietsch, W. 1991, A\&A 246, L36

Burwitz V. et al. 1995, A\&A (in press)

Campbell, C.G. 1985, MNRAS 215, 509

Chanmugam, G. 1992, ARA\&A 30, 143

Cropper, M. \& Wickramasinghe, D.T. 1993, MNRAS 260, 696

Cropper, M. et al. 1988, MNRAS 236, 29P 
Cropper, M., Mason, K.O. \& Mukai, K. 1990a, MNRAS 243, 565

Cropper, M. et al. 1990b, MNRAS 245, 760

Ferrario, L. et al. 1992, MNRAS 256, 252

Ferrario, L., Bailey, J. \& Wickramasinghe, D.T. 1993, MNRAS 262, 285

Gänsicke, B., Beuermann, K. \& de Martino, D. 1995, A\&A (submitted)

Garnavich, P.M. et al. 1995, ApJ (in press)

Haberl, F. et al. 1994, A\&A 291, 171

Hameury, J.M., King, A.R. \& Lasota, J.P. 1986, MNRAS 218, 695

Hameury, J.M., King, A.R. \& Lasota, J.P. 1988, MNRAS 237, 48

King, A.R. \& Lasota, J.P. 1979, MNRAS 188, 653

King, A.R. \& Whitehurst, R. 1991, MNRAS 250, 152

King, A.R., Frank, J. \& Ritter, H. 1985, MNRAS 213, 181

Kuijpers, J. \& Pringle, J.E. 1982, A\&A 114, L4

Lamb, D.Q. \& Masters, R. 1979, ApJ 234, L117

Mason, K.O. et al. 1992, MNRAS 258, 749

Morfill, G.E. et al. 1984, A\&A 137, 7

Östreicher, R. et al. 1990, ApJ 350, 324

Pasquini, L., Belloni, T. \& Abbott, T.M.C. 1994, A\&A 290, L17

Piirola, V., Hakala, P. \& Coyne S.J., G.V. 1993, ApJ 410, L107

Piirola, V. et al. 1994, A\&A 283, 163

Putney, A. \& Jordan, S. 1994, ApJ (in press)

Reinsch, K. et al. 1994, A\&A 291, L27

Ritter, H. \& Kolb, U. 1993, in $X$-ray Binaries, W.H.G. Lewin, J. van Paradijs \& E.P.J. van den Heuvel (Eds.), Cambridge Univ. Press, (in press)

Robinson, C.R. \& Córdova, F. 1994, ApJ (in press)

Romani, R. 1993, Nat 347, 741

Schmidt, G.D., Stockman, H.S. \& Grandi, S.A. 1983, ApJ 271, 735

Schmidt, G.D., Liebert, J. \& Stockman, H.S. 1994, ApJ (in press)

Schwope, A.D. 1991, PhD thesis, Technical University of Berlin

Schwope, A.D. \& Beuermann, K. 1990, A\&A 238, 173

Schwope, A.D., Beuermann, K. \& Jordan, S. 1995, A\&A (in press)

Schwope, A.D., Beuermann, K. \& Thomas, H.-C. 1990, A\&A 230, 120

Schwope, A.D., Thomas, H.-C. \& Beuermann, K. 1993a, A\&A 271, L25

Schwope, A.D. et al. 1993b, A\&A 278, 487

Schwope, A.D. et al. 1994, A\&A (in press)

Shafter, A.W. et al. 1995, ApJ (in press)

Silber, A. et al. 1992, ApJ 389, 704

Van Teeseling, A., Heise, J. \& Paerels, F. 1994, A\&A 281, 119

Verbunt, F. \& Zwaan, C. 1981, A\&A 100, L7

Walter, F.M., Wolk, S.J. \& Adams, N.R. 1995, ApJ (in press)

Watson, M.G. et al. 1989, MNRAS 237, 299

Wickramasinghe, D.T. \& Wu, K. 1991, MNRAS 253, 11P

Wickramasinghe, D.T. \& Wu, K. 1994, MNRAS 266, L1

Wickramasinghe, D.T., Ferrario, L. \& Bailey, J. 1989, ApJ 342, L35

Woelk, U. 1995, (in preparation)

Woelk, U. \& Beuermann, K. 1992, A\&A 256, 498

Woelk, U. \& Beuermann, K. 1993, A\&A 280, 169

Wu, K. \& Wickramasinghe, D.T. 1993, MNRAS 260, 141

Young, P., Schneider, D.P. \& Shectman, S.A. 1981, ApJ 245, 1043 